\title{
Reabsorção radicular patológica em molar decíduo submetido a tratamento endodôntico com pasta à base de Cloranfenicol, Tetraciclina e Óxido de Zinco \\ (CTZ): Relato de caso
}

Pathological root resorption in primary molar subject to endodontic treatment with paste based on chloramphenicol, tetracycline and zinc oxide (CTZ): Case report

Reabsorción patológica radicular en molar decidual sujeito a tratamiento endodóntico con pasta a base de cloranfenicol, tetraciclina y óxido de zinc (CTZ): Reporte de caso

Carlos Vinícius Ramos Leão de Oliveira ORCID: https://orcid.org/0000-0001-7134-1011 Universidade Federal de Alagoas, Brasil E-mail: carlosviniciustlc@hotmail.com

Kelly Rodrigues Mota

ORCID: https://orcid.org/0000-0002-1656-608X Universidade Federal de Alagoas, Brasil E-mail: kellyrmota@outlook.com

Diogo Gomes Brandão

ORCID: https://orcid.org/0000-0001-6167-0399 Universidade Federal de Alagoas, Brasil

E-mail: diogo.brandao2009@hotmail.com

Gustavo Henrique Porto Oliveira ORCID: https://orcid.org/0000-0002-7796-5359 Universidade Federal de Alagoas, Brasil E-mail: gustavoportoh@outlook.com

Patricia Batista Lopes do Nascimento ORCID: https://orcid.org/0000-0002-2355-4810 Universidade Federal de Alagoas, Brasil E-mail: patnasci@hotmail.com

Priscylla Gonçalves Correia Leite de Marcelos ORCID: https://orcid.org/0000-0002-5623-6835 Universidade Federal de Alagoas, Brasil

E-mail: priscylla.marcelos@foufal.ufal.br

Daniela Maria Carvalho Pugliesi ORCID: https://orcid.org/0000-0002-7854-0416 Universidade Federal de Alagoas, Brasil E-mail: dpugliesi@hotmail.com

Pamella Recco Alvares ORCID: https://orcid.org/0000-0003-3396-9339 Universidade de Pernambuco, Brasil E-mail: pamellarecco@ hotmail.com Leopoldo Cosme Silva ORCID: https://orcid.org/0000-0002-5755-1933 Universidade Federal de Alagoas, Brasil E-mail: leopoldocosme@gmail.com

João Victor Farias da Silva

ORCID: https://orcid.org/0000-0001-8800-5540 Universidade Federal de Sergipe, Brasil E-mail: joaovictorfarias15@gmail.com Renata Matos Lamenha Lins ORCID: https://orcid.org/0000-0002-2968-6849 Universidade Federal de Minas Gerais, Brasil E-mail: renatamlamenha@gmail.com

Valdeci Elias dos Santos Junior ORCID: https://orcid.org/0000-0001-9748-5830 Universidade Federal de Alagoas, Brasil E-mail: valdeciodonto@gmail.com 


\begin{abstract}
Resumo
O presente estudo relata um caso de reabsorção patológica radicular em um molar decíduo tratado endodônticamente com pasta CTZ. Paciente do gênero masculino, 7 anos de idade, procurou atendimento, acompanhado por seus genitores, com a queixa principal de que o dente apresentava dor aguda. Durante a anamnese, o responsável relatou que o dente vinha apresentando dor espontânea que se intensificava durante a mastigação. Ao exame clínico não foi constatado quaisquer alterações em mucosa, gengiva e língua. Entretanto, verificou-se a presença de exposição da câmera pulpar do dente 55, sem indícios de polpa, configurando um quadro clínico de necrose. Ao realizar o exame radiográfico inicial foi possível verificar lesão de cárie extensa atingindo a região de cornos pulpares distais e raízes com comprimento maior que $1 / 3$ e coroa do dente sucessor ainda não totalmente formada. Constatada a viabilidade da manutenção do dente 55 na cavidade oral, instituiu-se o tratamento endodôntico através da técnica LSTR com a pasta antibiótica CTZ (cloranfenicol, tetraciclina, óxido de zinco) e restauração com resina composta. O paciente foi acompanhado por um período de 3 e 12 meses após o tratamento inicial. Em ambas as visitas, a restauração se apresentou hígida e satisfatória, contudo, foi possível notar aumento da reabsorção radicular lateral e de radiolucidez na região de furca. Após constatada tal reabsorção o dente teve sua indicação para a exodontia. A pasta CTZ pode estimular o aumento da velocidade de rizólise em dentes decíduos por meio de reabsorções radiculares patológicas. Porém, estudos longitudinais precisam ser conduzidos.
\end{abstract}

Palavras-chave: Dente decíduo; Criança; Pulpotomia; Odontopediatria; Endodontia.

\begin{abstract}
The present study reports a case of pathological root resorption in a primary molar treated endodontically with CTZ paste. A 7-year-old male patient sought care, accompanied by his parents, with the main complaint that the tooth had acute pain. During the anamnesis, the guardian reported that the tooth had been showing spontaneous pain that intensified during chewing. Clinical examination revealed no changes in the mucosa, gums and tongue. However, there was the presence of exposure of the pulp chamber of tooth 55, without evidence of pulp, configuring a clinical picture of necrosis. When performing the initial radiographic examination, it was possible to verify extensive caries lesion reaching the region of distal pulp horns and roots greater than $1 / 3$ and the crown of the successor tooth not yet fully formed. Once the viability of maintaining tooth 55 in the oral cavity was verified, endodontic treatment was instituted using the LSTR technique with the CTZ antibiotic paste (chloramphenicol, tetracycline, zinc oxide) and restoration with composite resin. The patient was followed for a period of 3 and 12 months after the initial treatment.. In both visits, the restoration was healthy and satisfactory, however, it was possible to notice an increase in lateral root resorption and radiolucency in the furcation region. After confirming such resorption, the tooth had its indication for extraction. CTZ paste can stimulate the increase in rhizolysis speed in primary teeth through pathological root resorption. However, longitudinal studies need to be conducted.
\end{abstract}

Keywords: Deciduous tooth; Child; Pulpotomy; Pediatric dentistry; Endodontics.

\title{
Resumen
}

El presente estudio reporta un caso de reabsorción patológica radicular en un molar deciduo tratado endodónticamente con pasta CTZ. Un paciente de sexo masculino de 7 años buscó atención, acompañado de sus padres, con la queja principal de que el diente presentaba dolor agudo. Durante la anamnesis, el tutor informó que el diente había estado mostrando un dolor espontáneo que se intensificó durante la masticación. El examen clínico no reveló cambios en las mucosas, encías y lengua. Sin embargo, existió la presencia de exposición de la cámara pulpar del diente 55, sin evidencia de pulpa, configurando un cuadro clínico de necrosis. Al realizar el examen radiográfico inicial se pudo constatar que la lesión de caries extensa alcanzaba la región de los cuernos pulpares distales y raíces mayores de $1 / 3$ y la corona del diente sucesor aún no estaba completamente formada. Una vez comprobada la viabilidad de mantener el diente 55 en la cavidad oral, se instituyó el tratamiento endodóntico mediante la técnica LSTR con la pasta antibiótica CTZ (cloranfenicol, tetraciclina, óxido de zinc) y la restauración con resina compuesta. El paciente fue seguido durante un período de 3 y 12 meses después del tratamiento inicial. En ambas visitas, la restauración fue sana y satisfactoria, sin embargo, se pudo notar un aumento en la reabsorción radicular lateral y radiolucidez en la región de furca. Después de confirmar dicha reabsorción, el diente tenía su indicación de extracción. La pasta CTZ puede estimular el aumento de la velocidad de rizolisis en dientes temporales a través de la reabsorción radicular patológica. Sin embargo, es necesario realizar estudios longitudinales.

Palabras clave: Diente primario; Niño; Pulpotomía; Odontología pediatrica; Endodoncia.

\section{Introdução}

As principais alterações patológicas que acometem a polpa e os tecidos perirradiculares são de natureza inflamatória e de etiologia infecciosa, sendo que a cárie e a infecção do sistema de canais radiculares representam as principais fontes de 
agressão microbiana (Lima et al., 2015). A cárie dentária é uma doença multifatorial, progressiva mais comum na infância, na qual fatores biológicos, comportamentais e socioeconômicos são associados à sua ocorrência (Smail-Faugeron et al., 2018).

A rápida progressão das lesões de cárie em dentes decíduos, justificada pela diferença de composição tecidual em comparação com os permanentes e pela inabilidade quanto à higiene oral na infância, propiciam o acometimento, por vezes precoce, da polpa dental, gerando dor e impactos negativos à qualidade de vida das crianças (Chaffee et al., 2017). A alta prevalência de cárie ainda é um dos grandes problemas de saúde pública brasileira (Molina-Frechero et al., 2015), pois é uma das principais causadoras da perda de elementos dentários (Santos Junior et al., 2014).

É de suma importância para a saúde sistêmica e bem-estar biopsicossocial da criança manter os dentes decíduos na cavidade oral até sua esfoliação geneticamente programada, já que estes participam de funções importantes, tais como: fonação, mastigação e manutenção de espaço para a correta erupção dos sucessores permanentes (Nobrega et al., 2018).

É notório a evolução da Endodontia na Odontopediatria nos últimos anos (Aragão et al., 2020), no entanto, realizar o preparo mecânico em dentes decíduos ainda é um grande desafio devido peculiaridades anatômicas as quais sujeitam os profissionais a iatrogenias (Dantas-Neta et al., 2016). Desse modo, a instrumentação dos canais radiculares por meio do uso de limas endodônticas tem sido revista em dentes decíduos, pois, não só pode lesar o germe do dente permanente em formação, como também não garante a completa desinfecção do complexo sistema de canais radiculares dos dentes decíduos (Fernandes et al., 2020).

Devido a essa problemática, o conceito de Lesion Sterilization and Tissue Repair (LSTR) tem sido pautado como alternativa para a terapia pulpar em dentes decíduos desvitalizados, técnica essa que dispensa o preparo mecânico e se embasa pela utilização de combinações de agentes antimicrobianos de largo espectro de atuação (Tasanarong et al., 2016). Desenvolvida por Soller e Cappiello em 1959, a pasta CTZ composta de Cloranfenicol, Tetraciclina e Óxido de Zinco e eugenol tem se destacado pelo alto índice de sucesso clínico e radiográfico reportado (entre 81,8-100\% e 63-93\%, respectivamente) (Dantas-Neta et al 2016; Lokade et al., 2019) e pela facilidade de execução e redução do tempo clínico, especialmente vantajoso em pacientes não colaborativos (Santos Junior et al., 2017)

A pasta CTZ tem mostrado biocompatibilidade em tecido subcutâneo em modelo animal, no entanto, devido à morfologia complexa dos sistemas de canais, irregularidade do ápice radicular, processo de rizólise e proximidade com o germe do permanente ainda restam lacunas científicas a respeito dessa propriedade em humanos (Lima et al 2015; Moura Et al., 2018). Possíveis efeitos adversos, como a indução de reabsorção radicular patológica e/ou aceleração do processo de rizólise ainda precisam ser mais investigados.

Assim, este estudo se constitui em um relato de caso de um dente molar decíduo acometido por reabsorção patológica radicular após tratamento endodôntico com pasta CTZ.

\section{Metodologia}

Este estudo possui caráter observacional, qualitativo, descritivo, do tipo relato de caso (Pereira et al., 2018), no qual foi investigado um dente molar decíduo afetado por reabsorção patológica radicular e acentuado processo de rizólise após tratamento endodôntico com pasta CTZ.

Esse estudo foi aprovado pelo Comitê de Ética em Pesquisa da Universidade Federal de Alagoas - UFAL (Parecer 2.970.527/2018), obedecendo a Resolução n. ${ }^{\circ}$ 466/2012 da Comissão Nacional de Ética em Pesquisa e em consonância com a Declaração de Helsinki. 
Foram obtidos os Termo de Consentimento Livre e Esclarecido (TCLE) e o Termo de Assentimento Livre Esclarecido (TALE), assinados respectivamente pelos pais e pela criança. O consentimento verbal da criança também foi obtido em cada intervalo de avaliação.

\section{Relato de Caso}

Paciente do gênero masculino, 7 anos de idade, compareceu à Clínica Odontológica Infantil da Universidade Federal de Alagoas - FOUFAL, acompanhado por seus genitores, com a queixa principal de que o dente apresentava dor aguda.

Durante a anamnese, o responsável relatou que vinha apresentando dor espontânea que se intensificava durante a mastigação. Questionada sobre estado de saúde geral do paciente, a responsável referiu que a criança não possuía qualquer comorbidade ou alergia a qualquer fármaco. Relato de caso

Ao exame clínico não foi constatado quaisquer alterações em mucosa, gengiva e língua. Durante a inspeção dental verificou-se uma pobre higiene oral e presença de lesões de cárie nos elementos 53, 55 e 64 . Com relação ao dente 55 verificou-se a presença de exposição da câmera pulpar, sem indícios de polpa, configurando um quadro clínico de necrose. Foi possível constatar a ausência de edemas, fístulas ou abscessos nos tecidos periodontais adjacentes a este dente. No entanto, a criança relatava dor espontânea, exacerbada ao toque. Após o exame radiográfico inicial (figura 1a) foi possível verificar lesão de cárie extensa atingindo a região de cornos pulpares distais e raízes com comprimento maior que 1/3 e coroa do dente sucessor ainda não totalmente formada. Ao analisar os tecidos ósseos perirradicular e periapical não foi constatada qualquer lesão e todas as raízes apresentavam-se em estado de rizólise fisiológica normal, sem qualquer evidência de reabsorções patológicas (interna ou externa).

Constatada a viabilidade da manutenção do dente 55 na cavidade oral, instituiu-se o tratamento endodôntico sem a realização do preparo mecânico através da técnica LSTR com a pasta antibiótica CTZ (cloranfenicol, tetraciclina, óxido de zinco). O CTZ foi manipulado em farmácia de manipulação (A fórmula®, Alagoas, Brasil) nas proporções 1:1:2 (cloranfenicol, tetraciclina, óxido de zinco) em formato de cápsulas devidamente porcionadas. Para a manipulação da pasta, ao conteúdo da cápsula foi adicionado uma gota de eugenol (aproximadamente 0,05mL), formando uma pasta arenosa.

Após a administração da anestesia local com solução anestésica de Lidocaína 2\% com Epinefrina 1:100.000 (Alphacaine- DFL®, Indústria E Comércio S/A, Rio de Janeiro, Rio de Janeiro, Brasil) o dente foi isolado usando um dique de borracha. Toda a cárie foi removida com instrumentos manuais. Em seguida a câmara pulpar foi acessada com uma ponta diamantada 1012 (KG Soren $\AA$ ) em alta velocidade. Posteriormente, usando uma broca carbide 329 (KG Sorenß) foram ampliadas as entradas dos canais a uma abertura de $1 \mathrm{~mm}$ de diâmetro e $2 \mathrm{~mm}$ de profundidade. Após essa etapa, os sistemas de canais foram irrigados com soro fisiológico e as paredes da câmera pulpar foram quimicamente limpas com ácido fosfórico a $35 \%$ de modo a remover a smear layer. Por fim, a pasta CTZ foi inserida em cada duto dos canais previamente preparados e uma restauração com resina composta foi realizada (Filtek Z-100®; 3M-ESPE - St. Paul, MN, USA).

O paciente foi acompanhado por um período de 3 e 12 meses após o tratamento inicial. Na primeira consulta de retorno (após 3 meses) foi realizado a anamnese direcionada ao dente em questão, na qual foi verificada a ausência de qualquer queixa de dor associada ou sinais clínicos de comprometimento de tecido de suporte, como fistula ou abscesso. Ao exame radiográfico (Figura 1B) notou-se o início de uma reabsorção radicular lateral na raíz palatina e aumento de radiolucidez na região de furca. Após 12 meses, o paciente novamente retornou sem qualquer queixa clinica de dor ou sinais de comprometimento de tecido de suporte, porém ao exame radiográfico foi verificada a presença de intensa reabsorção, sugestiva de reabsorção patológica (Figura 1C). Em ambos os seguimentos, a restauração em resina composta se apresentou hígida e clinicamente satisfatória. Após constata tal reabsorção o dente teve sua indicação para a exodontia. 
Figura 1 - Avaliações radiográficas ao exame inicial e nos períodos de retorno de 3 meses e 1 ano.
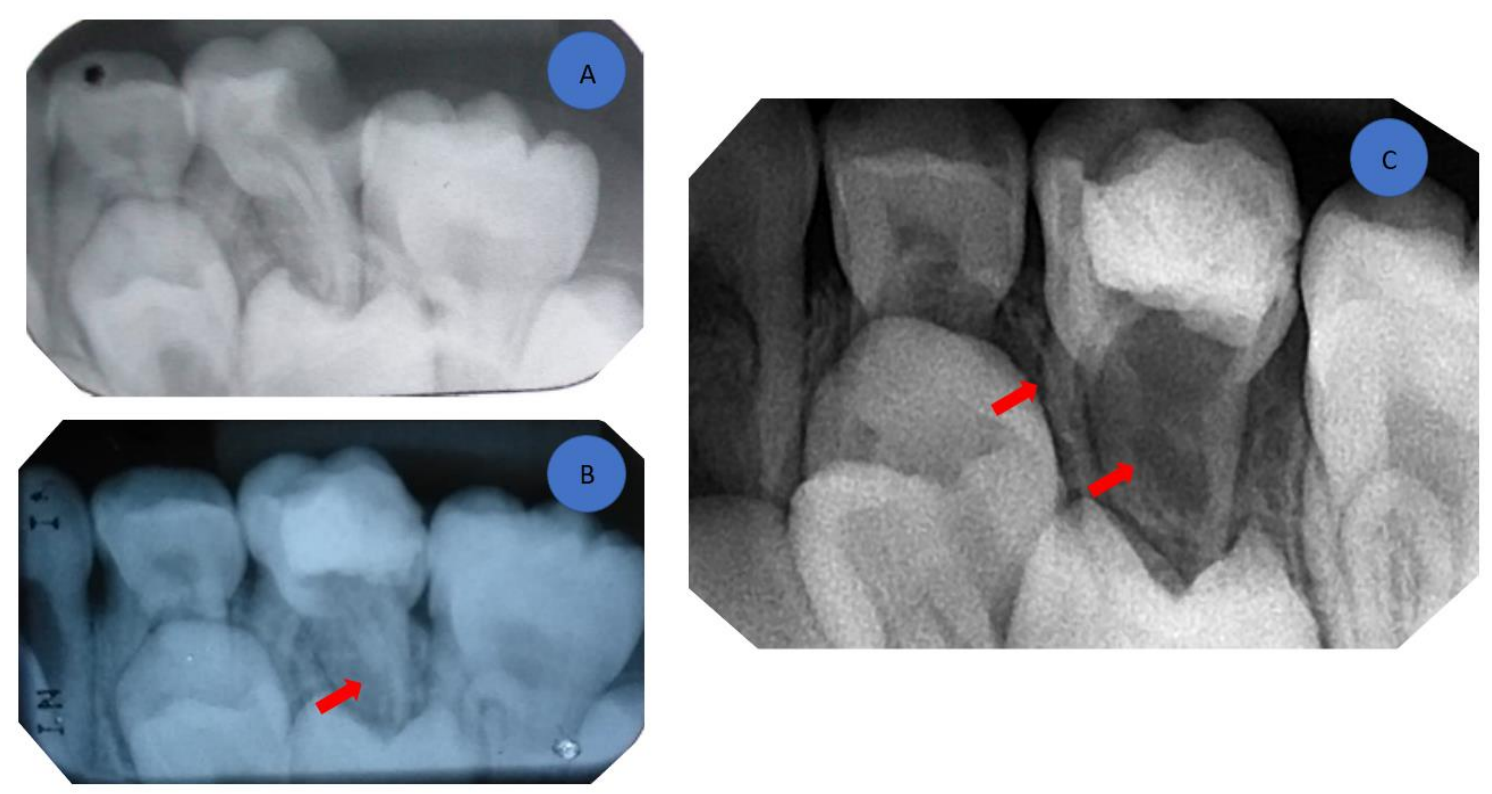

Legendas: (A) exame inicial; (B) Avaliação de 3 meses, mostrando o início de reabsorção patológica; (C) Avaliação após um ano, evidenciando extensa reabsorção patológica e aceleração da rizólise.

Fonte: Oliveira et al. (2020).

É interessante observar as áreas radiolúcidas sugestivas de reabsorção interna e externa indicadas nas radiografias através das setas vermelhas e o quão evidente foi a velocidade desse processo patológico entre os períodos observados.

\section{Discussão}

Os molares decíduos apresentam como característica um complexo sistema de canais radiculares e alta permeabilidade na região de furca (Fernandes. et al., 2020). Estes atributos anatômicos possibilitam que metabólitos oriundos da decomposição pulpar e/ou fármacos presentes nos materiais endodônticos possam desencadear reações inflamatórias locais. Tendo isso em vista, tais materiais devem possuir, além de propriedade antimicrobiana de amplo espectro de ação, uma elevada biocompatibilidade aos tecidos pulpares e perirradiculares, (Aragão, et al., 2020).

Neste sentido, a pasta CTZ se apresenta como uma alternativa viável para tal aplicabilidade, uma vez que possui biocompatibilidade aos tecidos orgânicos, assemelhando-se à difundida pasta de hidróxido de cálcio (Lima, et al., 2015). Embora a eficácia da pasta CTZ dependa de fatores como um correto diagnóstico, condição sistêmica, colaboração do paciente, anatomia interna do dente e resistência bacteriana (Fernandes. et al. 2020), este material promove a redução de monócitos, eosinófilos e células fagocitárias, o que vem a elucidar seu mecanismo de ação capaz de controlar infecções de origem endodônticas e propiciar a reparação tecidual local (Moura et al., 2018).

Esta redução em termos de inflamação na região perirradicular é tida como uma ação necessária para a manutenção dos tecidos dentários e periodontais em estado de higidez, haja vista que manutenção de um infiltrado inflamatório rico em células osteorremodeladoras e citocinas pró-inflamatórias é tido como um fator de risco para o surgimento de processos patológicos que promovam a reabsorção tecidual óssea e dentária (Singh et al., 2017; Travassos et al., 2020).

É notório que a reabsorção radicular em dentes decíduos é considerada fisiológica, contudo, mudanças em sua velocidade, padrão ou localização podem caracterizá-la como patológica (Gherghe et al., 2020). No caso em questão, a localização lateral dos achados radiolúcidos, após a aplicação do CTZ, foi considerada atípica, sendo indicativo para reabsorção patológica. Essa falha corrobora com os estudos de Oliveira \& Costa (2006) e Daher et al. (2015) em que foi 
evidenciada reabsorção radicular externa e lesão óssea perirradicular como principais achados radiográficos insatisfatórios em relação ao uso da pasta CTZ.

Ao analisar o caso supra relatado, verifica-se um amplo processo de reabsorção radicular externa e interna o qual levou a indicação de exodontia do dente envolvido. Essa tomada de decisão se procedeu pelos riscos inerentes à formação do esmalte do sucessor permanente (Sousa et al., 2020). Alterações nos padrões metabólicos, decorrentes do pH ácido da inflamação pode determinar uma condição chamada hipoplasia de Turner, caracterizada por defeito de esmalte em diferentes níveis a depender da severidade e do tempo de instalação do processo inflamatório irritante (Santos Júnior et al., 2013). Ademais, ainda há a associação entre os estágios iniciais de desenvolvimento dentário e sua predisposição a distúrbios da amelogênese quando exposto a fatores de risco (Sousa et al.,2020). Não obstante, os dentes superiores são mais propensos a defeitos hipoplásicos, visto que a baixa densidade do osso alveolar da maxila pode intensificar a difusão dos metabólitos inflamatórios até o dente sucessor em desenvolvimento. Logo, levando-se em consideração que o paciente possuía apenas 7 anos de idade, alterações no esmalte do pré-molar superior poderia ocorrer uma vez que a calcificação desse elemento se perdura até os doze anos de idade (Grossman,1986; Conchie, Munrou \& Andersom, 1970)

Segundo o guia de terapia pulpar para dentes decíduos da American Academy of Pediatric Dentistry (AAPD), a LSTR é recomendada para dentes que estão em processo de rizólise, justificando, dessa forma, o seu uso neste estudo. Entretanto, essa informação precisa ser vista com parcimônia, pois pulpectomias tradicionais que se utilizam de ação química-mecânica têm maiores chances de sucesso em terapias pulpares (Fonseca et al., 2020; Moghaddam, Mehran \& Zadeh, 2009; Pinheiro et al., 2014; Ramezanali et al., 2015). A associação de instrumentos específicos e agentes irrigantes/medicamentoso tem capacidade remover o biofilme bacteriano, evitar infecções endodônticas persistentes e, consequentemente, reabsorções patológicas (Neelakantan et al., 2017).

Este estudo apresenta limitações metodológicas inerente a um relato de caso, como um número baixo de individuo e variabilidade individual dos dados apresentados. No entanto, tal método é particularmente útil para descrever relatos raros ou poucos difundidos na literatura, como falhas de materiais ou desfechos inesperados como apresentados por esse caso. Estudos longitudinais precisam ser conduzidos para se obter evidência de qualidade a respeito da efetividade e segurança da pasta CTZ.

\section{Conclusão}

A pasta CTZ pode estimular o aumento da velocidade de rizólise em dentes decíduos por meio de reabsorções radiculares patológicas. Porém, estudos longitudinais, como ensaios clínicos randomizados, precisam ser conduzidos.

\section{Referências}

American Academy of Pediatric Dentistry. Pulp therapy for primary and immature permanent teeth. The Reference Manual of Pediatric Dentistry. Chicago, Ill.: American Academy of Pediatric Dentistry; 2020:384-92

Aragão, A. C., Pintor, A., Marceliano-Alves, M., Primo, L. G., Silva, A., Lopes, R. T., \& Neves, A. A. (2020). Root canal obturation materials and filling techniques for primary teeth: In vitro evaluation in polymer-based prototyped incisors. International journal of paediatric dentistry, 30(3), 381-389. https://doi.org/10.1111/ipd.12604

Chaffee, B. W., Rodrigues, P. H., Kramer, P. F., Vítolo, M. R., \& Feldens, C. A. (2017). Oral health-related quality-of-life scores differ by socioeconomic status and caries experience. Community dentistry and oral epidemiology, 45(3), 216-224. https://doi.org/10.1111/cdoe.12279 
Conchie, J. M., Munroe, J. D., \& Anderson, D. O. (1970). The incidence of staining of permanent teeth by the tetracyclines. Canadian Medical Association journal, 103(4), 351-356.

Daher, A., Viana, K. A., Leles, C. R. \& Costa, L. R. (2015). Ineffectiveness of Antibiotic-Based Pulpotomy for Primary Molars: a Survival Analysis. Brazilian Research in Pediatric Dentistry and Integrated Clinic, 15(1), 205-215. http://dx.doi.org/10.4034/PBOCI.2015.151.22

Dantas-Neta, N. B., Moura, L. F., Cruz, P. F., Moura, M. S., Paiva, S. M., Martins, C. C. \& Lima, M. D. (2016). Impact of molar-incisor hypomineralization on oral health-related quality of life in schoolchildren. Brazilian oral research, 30(1), e117. https://doi.org/10.1590/1807-3107BOR-2016.vol30.0117

Fernandes, M. L. F., Maia, C. A., Santos, A. M. C., Vilela, C. R., Araújo, F. R., Mohallen, M. L., Silveira, L. B. \& Fernandes, A. M. (2020). Antimicrobial Photodynamic Therapy in the Endodontic Treatment of Deciduous Teeth: In Vivo Pilot Study. Pesquisa Brasileira em Odontopediatria e Clínica Integrada, 20:e5309 https://doi.org/10.1590/pboci.2020.111

Fonseca, L. de A., Cangussu, R. A., Oliveira, A. S. de, Pinheiro, S. L., Shitsuka, C., \& Duarte, D. A. (2020). Comparação da desinfecção endodôntica de canais radiculares de dentes decíduos utilizando sistemas rotatório e reciprocante: Estudo in vitro. Research, Society and Development, 9(8), e457985882. https://doi.org/10.33448/rsd-v9i8.5882

Geibel, M. A., Carstens, S., Braisch, U., Rahman, A., Herz, M. \& Jablonski-Momeni, A. (2017). Radiographic diagnosis of proximal caries-influence of experience and gender of the dental staff. Clinical oral investigations, 21(9), 2761-2770. https://doi.org/10.1007/s00784-017-2078-2

Grossman, E. R. (1986). Tetracycline and staining of the teeth. JAMA, 255(18), 2442-2443. https://doi.org/10.1001/jama.1986.03370180067011

Lima, C. C., Conde Júnior, A. M., Rizzo, M. S., Moura, R. D., Moura, M. S., Lima, M. D., \& Moura, L. F. (2015). Biocompatibility of root filling pastes used in primary teeth. International endodontic journal, 48(5), 405-416. https://doi.org/10.1111/iej.12328

Lokade, A., Thakur, S., Singhal, P., Chauhan, D., \& Jayam, C. (2019). Comparative evaluation of clinical and radiographic success of three different lesion sterilization and tissue repair techniques as treatment options in primary molars requiring pulpectomy: An in vivo study. Journal of the Indian Society of Pedodontics and Preventive Dentistry, 37(2), 185-191. https://doi.org/10.4103/JISPPD.JISPPD_6_19

Moghaddam, K. N., Mehran, M., \& Zadeh H. F. (2009). Root Canal Cleaning Efficacy of Rotary and Hand Files Instrumentation in Primary Molars. Int Endod $J, 2(4), 53-7$

Molina-Frechero, N., Durán-Merino, D., Castañeda-Castaneira, E., \& Juárez-López, M. L. (2015). La caries y su relación con la higiene oral en preescolares mexicanos [Dental caries experience and its relation to oral hygiene in Mexican children]. Gaceta medica de Mexico, 151(4), 485-490.

Moura, L., Lima, M., Lima, C., Bandeira, A., Moura, M. S., Conde Júnior, A. M., \& Rizzo, M. (2018). Cellular profile of primary molars with pulp necrosis after treatment with antibiotic paste. International journal of experimental pathology, 99(5), 264-268. https://doi.org/10.1111/iep.12292

Neelakantan, P., Romero, M., Vera, J., Daood, U., Khan, A., Yan, A., \& Cheung, G. (2017). Biofilms in Endodontics-Current Status and Future Directions. International Journal of Molecular Sciences, 18(8), 1748:1-21. https://doi.org/10.3390/ijms18081748

Nobrega, M. L., Barbosa, C. C. N., \& Brum S. C. (2018) Implicações da perda precoce em odontopediatria. Revista Pró-UniverSUS, Jan./Jun.; 09 (1): $61-67$.

Oliveira, M. A. C., \& Costa, L. R. R. S., (2006) Clinical outcomes of CTZ pulpotomies in deciduous molars: A retrospectiv study. Robrac, 15 (40).

Pinheiro, S. L., da Silva, J. N., Gonçalves, R. O., \& Villalpando K. T. (2014). Manual and Rotary Instrumentation Ability to Reduce Enterococcus faecalis Associated with Photodynamic Therapy in Deciduous Molars. Braz Dent J, 25(6), 502-7.

Pereira A. S., et al. (2018). Metodologia da pesquisa científica. [e-book]. Santa Maria. Ed. UAB/NTE/UFSM. https://repositorio.ufsm.br/bitstream/handle/1/15824/Lic_Computacao_Metodologia-Pesquisa-Cientifica.pdf?s equence=1 
Research, Society and Development, v. 10, n. 1, e0810111347, 2021 (CC BY 4.0) | ISSN 2525-3409 | DOI: http://dx.doi.org/10.33448/rsd-v10i1.11347

Ramezanali, F., Afkhami, F., Soleimani, A., Kharrazifard, M. J., \& Rafiee, F. (2015). Comparison of Cleaning Efficacy and Instrumentation Time in Primary Molars: Two Rotary Instruments vs. Hand K-Files. Iran Endod J, 10(4), 240-3.

Santos Junior, V. E., Alencar Filho, A. V., Leite, A. C. G. L. \& Rosenblatt, A. (2013). Is There an Association Between Enamel Stains in Premolars and Endodontic Treatment of Their Primary Molar Predecessors? Pesq Bras Odontoped Clin Integr, 13(1), 17-21. http://dx.doi.org/10.4034/pboci.v13i1.1423

Santos Junior, V. E., de Sousa, R. M., Oliveira, M. C., de Caldas Junior, A. F., \& Rosenblatt, A. (2014). Early childhood caries and its relationship with perinatal, socioeconomic and nutritional risks: a cross-sectional study. BMC oral health, 14, 47. https://doi.org/10.1186/1472-6831-14-47

Smaïl-Faugeron, V., Glenny, A. M., Courson, F., Durieux, P., Muller-Bolla, M., \& Fron Chabouis, H. (2018). Pulp treatment for extensive decay in primary teeth. The Cochrane database of systematic reviews, 5(5), CD003220. https://doi.org/10.1002/14651858.CD003220.pub3

Singh,O., Sinha, D.J., Tyagi,S.P. \& Tyagi N. (2017) Root Resorption: Challenge to the Endodontist. International Journal of Dental Research and Oral Sciences 2.1, 31-41.

Sousa, H. C. S., Lima, M. D. M., Lima, C. C. B., Moura, M. S., Bandeira, A. V. L., Deus Moura, L. F. A. (2020). Prevalence of enamel defects in premolars whose predecessor were treated with extractions or antibiotic paste. Oral health and preventive dentistry. 18(4), 793-798.

Tasanarong, P., Dechatiwongse Na Ayudhya, T., Techanitiswad, T., \& Koontongkaew, S. (2016). Reduction of viable bacteria in dentinal tubules treated with a novel medicament (Z-Mix). Journal of dental sciences, 11(4), 419-426. https://doi.org/10.1016/j.jds.2016.06.006

Travassos, R. M. C., Negreiros, J. H. C. N., Ribeiro, Y. de A. T., Silva, Y. C. de L., Silva, M. C. de A. J., Souza, T. G. dos S., Barbosa, L. M., \& Silva, H. J. (2020). Sucesso no tratamento endodôntico em incisivo lateral superior com reabsorção radicular interna inflamatória não perfurante: relato de caso. Research, Society and Development, 9(9), e233997046. https://doi.org/10.33448/rsd-v9i9.7046 